\title{
IDENTIFICAÇÃO AUTOMÁTICA DAS ORDENS DOS MODELOS GARCH UTILIZANDO REDES NEURAIS
}

\author{
André Machado Caldeira, $M S c^{l}$ \\ Reinaldo Castro Souza, $\mathrm{PhD}^{2}$ \\ Maria Augusta Soares Machado, DSc ${ }^{3}$
}

\begin{abstract}
Resumo: Os modelos da família GARCH têm sido utilizados em larga escala para modelagem da volatilidade de ativos financeiros sendo o GARCH $(1,1)$ o mais utilizado na pratica. A fase de identificação das ordens dos modelos GARCH não tem sido explorada. Por outro lado, a tecnologia de sistemas especialistas tem sido utilizada em modelos de séries temporais como, por exemplo, em problemas de classificação de series temporais (Reynolds et al, 1995) e na identificação de modelos ARMA (Machado, 2000). O objetivo deste artigo é propor um sistema inteligente que seja capaz de identificar corretamente a ordem dos modelos GARCH, facilitando assim a escolha do modelo a ser utilizado e com isso evitar o uso indiscriminado do modelo GARCH $(1,1)$.
\end{abstract}

Palavras-chave: GARCH, Volatilidade, Identificação.

Abstract: GARCH models are being largely used to model the volatility of financial assets, and GARCH $(1,1)$ is the one mostly used. The identification of the model specification has not been fully explored. However, technology of specialist systems has been used in some applications of time series model as, for example, in time series classification problems (Reynolds et al, 1995) and ARMA models identification (Machado, 2000). The aim of this paper is to propose an intelligent system that can correctly identify the specification of GARCH models providing the right choose of the model to be used, avoiding the indiscriminate usage of GARCH $(1,1)$ model.

Key-words: GARCH, Volatility, Identification.

\section{INTRODUÇÃO}

"A identificação da ordem de um modelo GARCH, a ser ajustado a uma série real, usualmente é dificil. Recomenda-se que se use modelos de ordem baixa, como $(1,1),(1,2)$ ou $(2,1)$ e depois se escolha o modelo com base em vários critérios, como AIC ou BIC, ..." (Morettin e Toloi, 2004).

Os modelos ARCH e GARCH vêm sendo bastante explorados, tanto teoricamente quanto em aplicações práticas, desde suas respectivas criações em 1982 e 1986 respectivamente. Contudo a preocupação está muito concentrada, ou na captura dos fatos estilizados (a maioria dos estudos teóricos) ou previsão de volatilidade (que normalmente se assume o GARCH $(1,1)$ nos estudos aplicados a séries reais). Não existem muitos estudos concentrados na fase de identificação. Alguns estudos utilizando tecnologia de sistemas especialistas foram feitos em modelos de séries temporais como, por exemplo, em problemas de classificação de series temporais (Reynolds et al, 1995) e na identificação de modelos ARMA (Machado, 2000). Diante deste contexto, esse trabalho

PUC-RJ - amcaldeira@yahoo.com.br

PUC-RJ - reinaldo@ele.puc-rio.br

3 Ibmec-mmachado@ibmecrj.br 
tem como objetivo criar um sistema inteligente que seja capaz melhorar a fase de identificação da ordem dos modelos, evitando assim o uso indiscriminado do modelo GARCH $(1,1)$. Para isso, serão utilizadas séries temporais simuladas para a validação do sistema. Os resultados obtidos com o sistema inteligente serão comparados com a escolha dos modelos pelas estatísticas AIC e BIC.

Este artigo está dividido em quatro partes adicionais, sendo a próxima parte um resumo dos principais conceitos relacionados ao artigo. A terceira parte apresenta os resultados da identificação utilizando o método de escolha pelos critérios AIC e BIC. A penúltima parte propõe um modelo que pode ser utilizado para estudar a identificação. A quinta, e última parte, apresenta as conclusões do trabalho.

\section{CONCEITOS}

\section{MODELOS GARCH}

O mercado financeiro na atualidade sofre grande influência dos acontecimentos diários. Analisando uma série de retornos financeiros, que pode ser usualmente representada como uma série temporal, que apresenta alternância entre períodos de grande e pequena flutuação dos valores em torno da sua média, formando "aglomerados", podemos definir volatilidade como "a variação, ao longo do tempo, da variância condicional de uma série temporal." (Veiga; Fernandes e Baidya, 1993)

Essa volatilidade é boa e benéfica para o mercado financeiro, pois o aumento da volatilidade interessa não apenas aos investidores e analistas do mercado, como também aos economistas e "policymakers". Entretanto, quando essa volatilidade é excessiva, ela afeta negativamente à economia, prejudicando o bom funcionamento do sistema financeiro.

A excessiva volatilidade pode interferir no mercado financeiro de diversas formas:

- Nos preços de ativos financeiros: Essa volatilidade pode afetar o nível de investimento, pois o risco seria maior, induzindo uma procura de outros ativos com menores riscos em outros mercados.

- Na taxa de juros: Tendo custos mais altos para obtenção de créditos ou empréstimos, para projetos de investimentos, o nível da economia pode cair.

- Na taxa de câmbio: Pode provocar uma redução significativa no volume de importação do país, se os preços dos bens importados e exportados aumentarem devido ao risco cambial, fazendo com que o consumo destes bens seja reduzido.

A Volatilidade dentro da economia e para o mercado financeiro é muito importante, por isso, sua estimação e previsão têm um tratamento diferenciado dos modelos clássicos de séries temporais (ARIMA de Box.\& Jenkins, 1976), pois os mesmos não reproduzem algumas características fundamentais (fatos estilizados), como a não normalidade condicional/incondicional e a variância condicional não constante ao longo do tempo.

Existem diversos tipos de modelos para modelagem da volatilidade, como o Alisamento Exponencial (EWMA), modelos com volatilidade estocástica (VE) e GARCH. Esse trabalho está concentrado nos modelos da família GARCH, para maiores detalhes sobre outros modelos leia Clark (1973), Taylor (1980, 1986 e 1994), Tauchen e Pitts (1983). Hull e White (1987) e Harvey et al. (1994).

Antes de iniciar com os conceitos dos modelos GARCH é importante conhecer alguns fatos estilizados das séries financeiras, com o objetivo de clarificar a inspiração que está por de trás desses modelos. Para maior aprofundamento desses fatos estilizados leia Bernardo e Fernandes (1999).

- Fato Estilizado 1 - Estacionariedade: as propriedades estatísticas da série são invariantes ao longo do tempo.

- Fato Estilizado 2 - Fraca/Nenhuma dependência linear e dependência não linear (efeito GARCH): a série normalmente é pouco ou não autocorrelacionada, mas a série do quadrado das observações é autocorrelacionada.

- Fato Estilizado 3: Não-normalidade: As distribuiçôes das séries financeiras geralmente apresentam caudas pesadas e presença de assimetria, fugindo da curva Gaussiana.

- Fato Estilizado 4: Existência de conglomerados de Volatilidade: A série finan- 
ceira costuma alternar entre períodos de alta volatilidade e períodos com baixa volatilidade, ou seja, a variância condicional da série varia ao longo do tempo.

Uma hipótese central na derivação do modelo para precificação de opções, sugerido por Black e Scholes (1973), é a suposição da série de retornos dos ativos ter distribuição log-normal com média e variância constante ao longo do tempo. Porem, ele foi fielmente utilizado até Mandelbrot (1963) e Fama (1963 e 1965) proporem, tanto as distribuiçóes de caudas pesadas, quanto os conglomerados de volatilidade. As características foram interpretadas como evidencia de volatilidade estocástica de ativos financeiros.

Para representar essas características há aproximadamente duas décadas os modelos da família GARCH têm sido muito utilizados na literatura de finanças, principalmente quando se fala em derivativos. $\mathrm{O}$ sucesso inicial dos modelos $\mathrm{ARCH}$ em capturar a dependência não linear permitiu muitas extensões.

\section{Representações dos modelos GARCH}

O primeiro modelo da família GARCH foi sugerido por Engle (1982), com o objetivo de captar parte dos fatos estilizados de séries financeiras, ele propôs modelar a série dos quadrados dos retornos por um modelo Autoregressivo de ordem q $(A R(q))$, chamando assim esse modelo de Autoregressive Conditional Heteroskedastic ou $\mathrm{ARCH}(\mathrm{q})$, que pode ser expresso pela expressão:

$$
u_{t}^{2}=\zeta+a_{1} u_{t-1}^{2}+a_{2} u_{t-2}^{2}+\ldots+a_{q} u_{t-q}^{2}+w_{t}
$$

onde $w_{t}$ é um ruído branco:

$$
\begin{aligned}
& E\left(w_{t}\right)=0 \\
& E\left(w_{t} w_{\tau}\right)= \begin{cases}\lambda^{2} & \text { para } \mathrm{t}=\tau \\
0 & \text { caso contrário }\end{cases}
\end{aligned}
$$

Às vezes é conveniente utilizar uma representação alternativa para o processo ARCH (q) que impõe uma hipótese um pouco mais forte a respeito da dependência serial de $u_{t}$, suponha que:

$$
u_{t}=\sqrt{h_{t}} v_{t}
$$

Onde:

$$
E\left(\mathrm{v}_{t}\right)=0 \quad E\left(\mathrm{v}_{t}^{2}\right)=1
$$

Se ${ }^{h}$ for escrito como:

$$
h_{t}=\zeta+a_{1} u_{t-1}^{2}+a_{2} u_{t-2}^{2}+\ldots+a_{q} u_{t-q}^{2}
$$

Implica em:

$$
E\left(u_{t}^{2} \mid u_{t-1}, u_{t-2}, \ldots\right)=\zeta+a_{1} u_{t-1}^{2}+a_{2} u_{t-2}^{2}+\ldots+a_{q} u_{t-q}^{2}
$$

Logo, se $u_{t}$ for gerado por e, então $u_{t}$ segue um processo ARCH(q), e se e forem substituídos em , o resultado é:

$$
h_{t} v_{t}=h_{t}+w_{t}
$$

Utilizando a especificação de, a inovação $w_{t}$ sobre a representação do $\mathrm{AR}(\mathrm{q})$ para $u_{t}^{2} \mathrm{em}$ pode ser expressa como:

$$
w_{t}=h_{t}\left(v_{t}^{2}-1\right)
$$

Note que a partir de, mesmo se a variância incondicional de ${ }^{w_{t}}$ foi assumida constante, a variância condicional de $w_{t}$ varia ao longo do tempo, assim, o modelo ARCH pode descrever os conglomerados de volatilidade.

Em 1986, Bollerslev notou em evidencias empíricas que era necessário estimar modelos $\mathrm{ARCH}$ com ordens muito altas para captar a dinâmica da variância condicional, com isso criou uma forma mais geral e parcimoniosa dos modelos $\mathrm{ARCH}$, chamando de Gereralized ARCH ou (GARCH) (Bollerslev, 1986) para solucionar esse problema.

A mesma idéia de parcimônia utilizada nos modelos ARMA foi utilizada nos modelos GARCH. Ou seja, demonstra-se facilmente que um MA de ordem um é equivalente a um AR de ordem infinita, então para reduzir o numero de parâmetros do modelo se utiliza a junção do AR com o MA, criando o ARMA. O modelo GAR$\mathrm{CH}$ é baseado na especificação do modelo ARCH de ordem infinita e ${ }^{h_{t}}$ pode ser expresso como:

$$
h_{t}=k+\sum_{i=1}^{q} \alpha_{t-i} u_{t-i}^{2}+\sum_{j=1}^{p} \beta_{t-j} h_{t-j}^{2}
$$

Da mesma forma que o modelo ARCH depende de restrições para $h_{t}$ ser positivo para todo $\mathrm{t}$, os modelos GARCH dependem das restrições de $k>0, \alpha_{i} \geq 0$ e $\beta_{j} \geq 0$. Nelson e Cao (1992) 
notaram que as condições $\alpha_{i} \geq 0$ e $\beta_{j} \geq 0$ eram suficientes, mas não necessárias, com isso argumentaram que impondo que todos os coeficientes sejam não negativos é muito restritivo e alguns desses coeficientes foram estimados negativos na pratica com a variância condicional positiva. Conseqüentemente, foi relaxada essa imposição, e na prática costuma-se estimar sem essas restrições.

Em muitas aplicações de séries temporais de alta freqüência, a variância condicional estimada usando um processo GARCH $(\mathrm{p}, \mathrm{q})$ exibe uma forte persistência, que é:

$$
\begin{aligned}
& \sum_{j=1}^{p} \beta_{j}+\sum_{i=1}^{q} \alpha_{i} \approx 1 \\
& \text { Se } \sum_{j=1}^{p} \beta_{j}+\sum_{i=1}^{q} \alpha_{i}<1 \text {, o processo }\left(u_{t}\right) \text { é }
\end{aligned}
$$
estacionário de segunda ordem, e o choque na variância condicional de $\sigma_{t}^{2}$ tem um impacto decrescente em $\sigma_{t+h}^{2}$, quando h cresce, e é assintoticamente insignificante. Essa propriedade é chamada na literatura de persistência.

Outras variações foram propostas com objetivos diversos como o Exponential GARCH (EGARCH) [Nelson, 1991 e Engle e Ng, 1993] e o TGARCH [Zakoian, 1991, Glosten et al., 1993, Rabemananjara e Zakoian, 1993] que buscam captar o efeito de assimetria, ou seja, o fato de choques negativos nos retorno dos ativos apresenta um impacto maior do que os positivos.

Os modelos ARCH e GARCH serão utilizados como objeto de estudo.

\section{Estratégia de Modelagem}

Segundo Franses e Djik (2000), a seqüência de modelagem normalmente envolve os seguintes passos:

- Calcular estatísticas de séries temporais (ACF e PACF)

- Comparar os valores ou tamanhos dessas estatísticas com valores teóricos que digam se o modelo é adequado. (Identificação)

- Estimar os parâmetros do modelo sugerido no passo 2. (Estimação)

- Avaliar modelos utilizando métricas de adequação. (Validação)

- Reespecificar o modelo se necessário.

- Use o modelo com propósitos descritivos ou preditivos. (Previsão)
- A preocupação deste estudo está na fase de identificação da estrutura de defasagem apropriada para a equação de variância condicional em um processo GARCH. As funções de autocorrelação e de autocorrelação parcial das séries de inovações são normalmente usadas para identificar e validar o comportamento temporal das séries dos modelos ARMA (Box and Jenkins, 1976). Bollerslev et al. (1988) mostrou que essas mesmas funçôes, quando aplicadas à série dos quadrados dos resíduos podem ser úteis para identificar e validar o comportamento temporal da equação da variância condicional na forma de GARCH. Logo, a identificação e validaçãao de um processo GARCH pode ser feita como:

Seja $\tau_{n}$ a n-ésima autocorrelação e $\phi_{k k}$ a k-ésima autocorrelação parcial de $u_{t}^{2}$ obtidos pela solução das equações para o GARCH, análogas as equações de Yule-Walker. As interpretações usuais são aplicáveis. Para um processo $\operatorname{ARCH}(q), \phi_{k k}$ tem um corte brusco após o q-ésimo lag, comportamento idêntico a função de autocorrelação parcial dos processos $A R(q)$. Por outro lado, a função de autocorrelação de $u_{t}^{2}$ para um processo $\operatorname{GARCH}(q, p)$ é em geral diferente de zero e dacai lentamente. Desta maneira, as funções de autocorrelação e autocorrelação parcial de $u_{t}^{2}$ podem ser utilizadas para identificar e validar a forma dos modelos GARCH.

Outra forma de identificar a arquitetura dos modelos GARCH é utilizar as estatísticas AIC e BIC, o modelo que apresentar a menor estatística é selecionado como o modelo identificado. $\mathrm{O}$ capítulo 3 apresenta alguns resultados utilizando essa forma de identificação.

Esse trabalho está concentrado na fase de identificação dos modelos, em particular da dos modelos GARCH simétricos.

\section{REDES NEURAIS}

Um dos modelos de inteligência computacional mais utilizados nos dias de hoje são as redes neurais. De acordo com Haykin (1999), em 1943, McCulloch e Pitts deram início à era moderna das redes neurais com um trabalho pioneiro e inovador. Ao aliar os conhecimentos de McCulloch, 
psiquiatra e neuroanatomista, com o raciocínio lógico do prodígio matemático Pitts, esse trabalho descreve um cálculo lógico das redes neurais, assumindo um modelo de um neurônio que seguia uma lei "tudo ou nada". Com um numero suficiente dessas unidades simples e com conexões sinápticas ajustadas apropriadamente e operando de forma síncrona, eles mostram que uma rede assim construída realizaria, a principio, a computação de qualquer função computável. Esse trabalho serviu de inspiração para diversos pesquisadores como Von Neumann, Hebb, Ashby, Taylor entre outros e é considerado o marco de inicio das disciplinas redes neurais e inteligência artificial.

As redes neurais são modelos matemáticos inspirados no cérebro humano, ou seja, são construídas a partir de arquiteturas de neurônios conectados, cada neurônio sendo representado por uma equação matemática, e cada ligação entre os neurônios sendo representadas por pesos ou funções, que geram sinais de propagação simulando a sinapse. A Figura 1 exemplifica uma arquitetura de uma rede neural de múltiplas camadas.

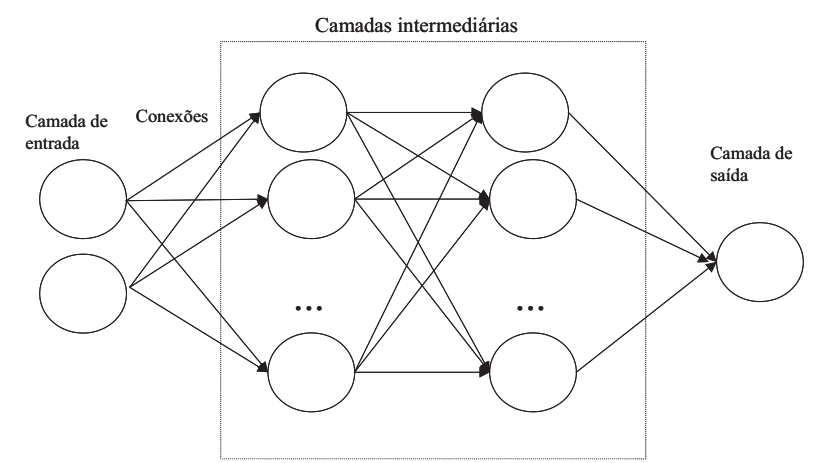

Figura 1: Exemplo de arquitetura de uma rede neural de múltiplas camadas

A camada de entrada representa os padrões (ou observações) que serão apresentados à rede. Essa camada é ligada na primeira camada intermediária por pesos, ou seja, o quanto determinado padrão vai influenciar cada neurônio da próxima camada. As camadas escondidas também estão ligadas por pesos sinápticos, o que pode ser interpretado também como suas contribuições para os próximos neurônios ligados. Esses pesos possuem o "conhecimento" da rede, pois são eles que são adaptados durante o treinamento. $\mathrm{Na}$ camada escondida é feita a maior parte do processamento. A camada de saída fornece o resultado de todos os cálculos entre valores de entrada, pesos e funções de ativação.
Cada neurônio (das camadas intermediarias e de saída) possui uma função de ativação, que dado o sinal recebido pelos outros neurônios, esse neurônio fica mais ativo ou não. O resultado dessa ativação é utilizado para passar o sinal para outro neurônio ou para o resultado final da rede. Uma rede neural é especificada principalmente pela sua topologia, pelas características dos neurônios e pelas regras de treinamento.

A maioria das redes neurais é treinada por algum algoritmo representado por uma equação matemática ou várias equações combinadas, com o objetivo de produzir, a partir da saída da rede, um valor numérico que servirá para ajustar o valor das conexôes de pesos de cada unidade de processamento. Esse algoritmo determina como os pesos de ligação da rede serão ajustados, com isso alterara a ativação dos neurônios diante de um conjunto específico de estímulos de entrada, o que pode ser chamado de aprendizado. Existem dois tipos de aprendizados para as redes neurais, o aprendizado supervisionado e o não supervisionado. No aprendizado supervisionado, também chamado de aprendizagem por correção de erro, os dados possuem exemplos, com isso se compara à saída da rede com os exemplos, ou seja, esses tipos de algoritmos visam minimizar esse erro. Já no aprendizado não supervisionado, também chamado de aprendizado baseado em memória, não existem exemplos a serem seguidos, e os algoritmos visam agrupar dados que possuem similaridades, e maximizar a diferença entre as características de grupos diferentes.

\section{IDENTIFICAÇÃO UTILIZANDO AIC E BIC}

Para testar a identificação dos modelos com base nos critérios AIC e BIC o primeiro passo foi simular séries temporais pelos modelos da família GARCH. O Matlab foi a ferramenta utilizada nesse estudo.

Os modelos utilizados para comparação foram: ARCH (1), ARCH (2), GARCH(1,1), GAR$\mathrm{CH}(2,1)$ e GARCH $(1,2)$, sendo simuladas 1.600 séries utilizando cada um dos modelos anteriores (variando o tamanho das séries, representando séries diárias de um mês (22), um trimestre (66), um semestre (132) e um ano (264), respectivamente), totalizando 8.000 séries. Para essa simulação foram 
gerados números aleatórios no intervalo $(0,1)$, respeitando as condições de estacionariedade dos modelos GARCH. Para os critérios de identificação foram escolhidos os modelos com a estrutura que apresentar a menor estatística AIC e BIC dentre os modelos estimados por máxima verossimilhança. A Tabela 1 apresenta uma análise de sensibilidade para os acertos da identificação dos modelos, quando se aumenta o tamanho das séries.

\section{Tabela 1: Resultados das identificações dos modelos pelos critérios AIC e BIC}

\begin{tabular}{|c|c|c|c|c|}
\hline \multirow{2}{*}{$\begin{array}{c}\text { Tamanho } \\
\text { das séries }\end{array}$} & \multicolumn{2}{|c|}{$\begin{array}{c}\text { Identificação } \\
\text { pelo AIC }\end{array}$} & \multicolumn{2}{c|}{$\begin{array}{c}\text { Identificação } \\
\text { pelo BIC }\end{array}$} \\
\cline { 2 - 5 } & Acertos & $\begin{array}{c}\% \text { de } \\
\text { acertos }\end{array}$ & Acertos & $\begin{array}{c}\% \text { de } \\
\text { acertos }\end{array}$ \\
\hline 22 & 488 & $24,4 \%$ & 465 & $23,3 \%$ \\
\hline 66 & 809 & $40,5 \%$ & 734 & $36,7 \%$ \\
\hline 132 & 1070. & $53,5 \%$ & 947 & $47,4 \%$ \\
\hline 264 & 1.371 & $68,6 \%$ & 1.200 & $60,0 \%$ \\
\hline Total & 3.738 & $\mathbf{4 6 , 7 \%}$ & 3.346 & $\mathbf{4 1 , 8 \%}$ \\
\hline
\end{tabular}

A identificação por esses critérios apresentou um acerto abaixo de $50 \%$ em média. Para as séries anuais, esse acerto foi melhor, alcançando $68,6 \%$. Porém, considerando as séries com menor número de observações (por exemplo, séries de empresas que fizeram IPO recentemente), a identificação fica muito mais baixa.

Observando o erro de identificação por cada modelo, apresentado na Tabela 2 e Tabela 3, pode ser observado que quanto maior a ordem do modelo, maior o erro de identificação, intuitivamente esse resultado já era esperado, uma vez que essas estatísticas buscam a parcimônia, ou seja, elas penalizam os modelos com ordens mais altas, tendendo a "viezar" a identificação para modelos com ordens mais baixas.

Como o critério AIC apresentou um melhor resultado de classificação, esse critério servirá como parâmetro de comparação com o modelo de identificação sugerido.

Tabela 2: Percentual de acerto na identificação por modelo pelo AIC

\begin{tabular}{|c|c|c|c|c|c|c|}
\hline $\mathbf{N}$ & ARCH (1) & ARCH (2) & GARCH (1,1) & GARCH (2,1) & GARCH (1,2) & Total \\
\hline 22 & $94,5 \%$ & $22,5 \%$ & $3,5 \%$ & $1,5 \%$ & $0,0 \%$ & $24,4 \%$ \\
\hline 66 & $92,8 \%$ & $64,8 \%$ & $29,5 \%$ & $8,0 \%$ & $7,3 \%$ & $40,5 \%$ \\
\hline 132 & $92,3 \%$ & $82,5 \%$ & $54,0 \%$ & $23,3 \%$ & $15,5 \%$ & $53,5 \%$ \\
\hline 264 & $93,0 \%$ & $89,8 \%$ & $74,5 \%$ & $53,0 \%$ & $32,5 \%$ & $68,6 \%$ \\
\hline Total & $93,1 \%$ & $64,9 \%$ & $40,4 \%$ & $21,4 \%$ & $13,8 \%$ & $46,7 \%$ \\
\hline
\end{tabular}

Tabela 3:Percentual de acerto na identificação por modelo pelo BIC

\begin{tabular}{|c|c|c|c|c|c|c|}
\hline $\mathbf{N}$ & ARCH(1) & ARCH(2) & GARCH(1,1) & GARCH(2,1) & GARCH(1,2) & Total \\
\hline 22 & $96,8 \%$ & $16,0 \%$ & $3,0 \%$ & $0,5 \%$ & $0,0 \%$ & $23,3 \%$ \\
\hline 66 & $98,8 \%$ & $56,5 \%$ & $25,0 \%$ & $2,0 \%$ & $1,3 \%$ & $36,7 \%$ \\
\hline 132 & $99,3 \%$ & $79,0 \%$ & $53,8 \%$ & $3,5 \%$ & $1,3 \%$ & $47,4 \%$ \\
\hline 264 & $99,5 \%$ & $91,5 \%$ & $83,0 \%$ & $19,8 \%$ & $6,3 \%$ & $60,0 \%$ \\
\hline Total & $98,6 \%$ & $60,8 \%$ & $41,2 \%$ & $6,4 \%$ & $2,2 \%$ & $41,8 \%$ \\
\hline
\end{tabular}




\section{MODELO DE REDES NEURAIS PARA AUXILIO À IDENTIFICAÇÃO}

Para verificar a oportunidade de identificação dos modelos GARCH utilizando técnicas de inteligência computacional, uma rede neural pode ser especificada conforme a estrutura apresentada na Figura 2.

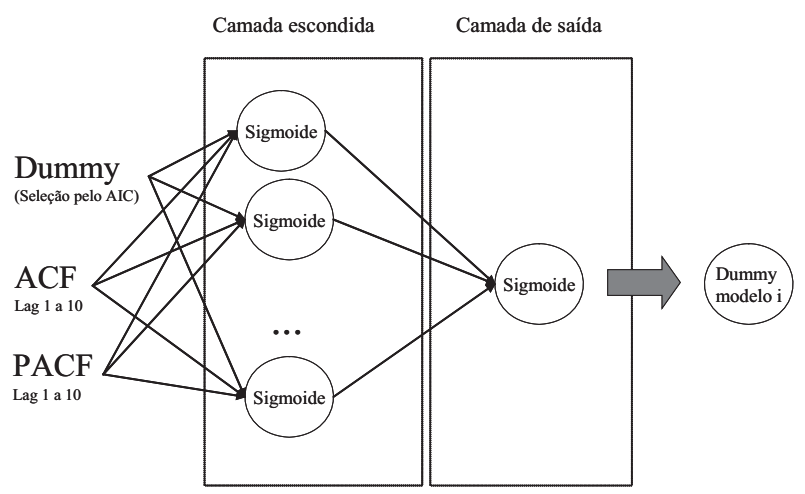

Figura 2: Estrutura da rede para identificação dos modelos GARCH

\section{CONSIDERAÇÔES FINAIS}

Como pode ser visto no capítulo 3, os critérios de informação AIC e BIC acertam apenas $46,7 \%$ e $41,8 \%$ dos casos, respectivamente, desconsiderando as séries anuais, performance de acertos é de 39,5\% e 35,8\% para os critérios AIC e BIC, respectivamente. Assim claramente existe oportunidade de aprimoramento na fase de identificação dos modelos GARCH.

Como próximos passos pretende-se implementar testar esse método de identificação para escolher modelos a serem aplicados para previsão da volatilidade de séries referente a ações de empresas que fizeram IPO recentemente. Essa aplicação será importante, uma vez que o mercado de ações brasileiro está se consolidando como uma boa opção de captação de recursos para empresas de médio e grande porte, esse tipo de série estará cada vez mais presente no mercado financeiro.

\section{REFERÊNCIAS}

BERNARDO, M. R. e FERNANDES, C. A. Utilização de Modelos não-lineares não-Gaussianos para Estimação de Volatilidade de Séries Temporais Financeiras. Dissertação (Graduação) - Pontifícia Universidade Católica do Rio de Janeiro, 1998.

BLACK F. \& SCHOLES, M. The pricing of options and corporate liabilities. Journal of Political Economy, 81, p. 637-59, 1973.

BOLLERSLEV, T. Generalized autorregressive conditional heteroskedasticity. Journal of Econometrics, v,31, p. 303-327, 1986.

BOLLERSLEV, T.; ENGLE, R. and WOOLDRIDGE, J. A capital asset pricing model with time varying covariances. Journal of Political Economy, v.96, p.116-131, 1988.

BOX, G. E. P. and JENKINS, G. M. Time Series Analysis: Forecasting and Control. Holden-Day, San Francisco, 1976.

CLARK, P. K. A subordinated Stochastic Process model with finite variance for speculative prices. Econometrica, 41, 135-155, 1973.

ENGLE, R. F. Autoregressive conditional heteroskedasticity with estimates of the variance of United Kingdom inflation. Econometrica, v.50, n.4, p.987-1007, 1982.

ENGLE, R. F. \& NG, V. K. Measuring and testing the impact of news on volatility. Journal of Finance, v. 48, p.1749-1778, 1993.

FAMA, E. Mandelbrot and the Stable Paretian hypothesis. Journal of Business, v.36, p.420-429, 1963.

FAMA, E. The behavior of stock prices, Journal of Business, v.47, p.244-280, 1965.

FRANSES, P. H.; DIJK, D. V. Nonlinear time series models in empirical finance. Cambridge University Press, 2000.

GLOSTEN, L., JAGANNATHAN, R. and RUNKLE, D. Relationship between the expected value and the volatility of the nominal excess return on stocks. Journal of Finance, v.48, p.17791801, 1993. 
HARVEY, A. C.; RUIZ, E. \& SHEPHARD, N. Modeling Stochastic variance models. Review of Economic Studies, 61, 247-267, 1994.

HAYKIN, S. Neural networks: a comprehensive foundation Prentice-Hall, 1999.

HULL, J. \& WHITE, W. The pricing of options on assets with stochastic volatility. Journal of Finance, v.42, p.281-300, 1987.

MACHADO, M. A. S. Auxílio à Idenficação de Modelo Box \& Jenkins Usando Redes Neurais Nebulosas. Pesquisa Naval, v.7, n.49, 2000.

MANDELBROT, B. The variation of certain speculative prices. Journal of Business, v.36, p.394-419, 1963.

MORETTIN, P. A.; TOLOI, C. M. C. Análise de séries temporais. Edgard Blücher, São Paulo, 2004 .

NELSON, D. Conditional heteroskedasticity in assets returns: a new approach. Econometrica, v.59, n.2, p.347-370, 1991.

NELSON, D. B. and CAO, C. Q. Inequality constraints in the univariate GARCH model. Journal of Business and Economic Statistics, v.10, p.229235, 1992.
RABEMANANJARA, R. and ZAKOIAN, J. M. Threshold ARCH models and asymmetries in volatility. Journal of Applied Econometrics, v.8, p.31-49, 1993.

REYNOLDS, B.; STEVENS, T.; MELLICHAMP, R.; SMITH, M. J. Box-Jenkins Forecast Model Identification. A. I. Expert, 1995.

TAUCHEN, G. E. \& PITTS, M. The price variability-volume relationship on speculative markets. Econometrica, v.51, p. 485-505, 1993.

TAYLOR, S. J. Conjectured models for trend in financial prices, tests and forecast. Journal of the Royal Sta, 1980.

TAYLOR, S. J. Modeling Financial Time Series". New York: John Wiley, 1996.

TAYLOR, S. J. Modeling Stochastic Volatility. Mathematical Finance, v.4, p.183-204, 1994.

VEIGA FILHO, A. L.; FERNANDES, C. A. C. \& BAIDYA, T. Medidas de Volatilidade para Opções. XXV SBPO/SOBRAPO, p.185-187, 1993.

ZAKOIAN, J. M. Threshold heteroskedastic models. Technical report, INSEE, 1991. 